\title{
Technè
}

La science au service de l'histoire de l'art et de la préservation des biens culturels

49 | 2020

Le mobilier Boulle

\section{Le nettoyage des bronzes dorés}

Cleaning gilt bronzes

\section{Emmanuel Plé et Julie Schröter}

\section{OpenEdition \\ Journals}

Édition électronique

URL : https://journals.openedition.org/techne/6188

DOI : 10.4000/techne.6188

ISSN : 2534-5168

\section{Éditeur}

C2RMF

\section{Édition imprimée}

Date de publication : 1 décembre 2020

Pagination : 117-121

ISBN : 978-2-11-152832-1

ISSN : $1254-7867$

\section{Référence électronique}

Emmanuel Plé et Julie Schröter, «Le nettoyage des bronzes dorés », Technè [En ligne], 49 | 2020, mis en ligne le 11 décembre 2020, consulté le 02 avril 2022. URL : http://journals.openedition.org/techne/ 6188 ; DOI : https://doi.org/10.4000/techne.6188

\section{(c) $(1)(9)$}

La revue Technè. La science au service de l'histoire de l'art et de la préservation des biens culturels est mise à disposition selon les termes de la Licence Creative Commons Attribution - Pas d'Utilisation Commerciale - Pas de Modification 4.0 International. 
Emmanuel Plé Julie Schröter

\section{Le nettoyage des bronzes dorés}

\author{
Cleaning gilt bronzes
}

Résumé. Cet article présente l'un des protocoles de nettoyage des bronzes dorés encrassés et ternis actuellement employés au C2RMF. Les avantages et limitations possibles du procédé sont évoqués à partir d'observations directement menées sur des auvres, ainsi qu'à partir de tests comparatifs simples effectués en atelier sur des coupons métalliques. Si les résultats obtenus permettent d'affiner les paramètres les plus adaptés en termes d'innocuité vis-à-vis des bronzes dorés, ce premier constat ouvre la perspective sur une étude plus approfondie prenant en compte d'autres variables et modes d'évaluation. Enfin, des améliorations de l'appareillage utilisé sont proposées afin d'assurer un meilleur contrôle du processus de nettoyage par le conservateur-restaurateur.

Mots-clés. Bronzes dorés, nettoyage, vapeur d'eau chaude, gommage.

\begin{abstract}
The paper presents one of the protocols for cleaning grimy, tarnished gilt bronzes currently employed at the C2RMF. The potential advantages and limitations of the process are recalled based on direct observations of the works, as well as simple comparative tests carried out on metal remnants in the workshop. While the results obtained enable us to refine the parameters best adapted to preventing damage to the gilt bronzes, this initial assessment opens the way for a more profound study that would take into account other variables and evaluation methods. Lastly, improvements could be made to the equipment to ensure better control and follow-up of the cleaning process by the conservator-restorer.
\end{abstract}

Keywords. Gilt bronzes, cleaning, steaming, scrubbing.

\section{Introduction}

Il existe un large choix de techniques de nettoyage pour les alliages de cuivre doré historiques. Le protocole dépend de l'état de surface de l'objet, du type et de la qualité du support, des éventuelles patines intentionnelles, de l'homogénéisation au sein d'une collection et/ou des matériaux composites constituant l'œuvre, du niveau de finition en fin de restauration...

Du fait de la forte valeur esthétique de ces bronzes décoratifs, l'opération de nettoyage a souvent pour objectif principal de mettre en valeur les décors et les éventuelles finitions de surface de la dorure. Les éléments concernés sont notamment des appliques de meubles, mais aussi des éléments de luminaires.

Depuis 2008, le protocole actuellement privilégié au sein du C2RMF pour le traitement de bronzes dorés encrassés et ternis est un démontage suivi d'un nettoyage à la vapeur d'eau chaude sous pression, puis d'un gommage des brunis. L'objet de cet article est de présenter la méthode appliquée, les essais simples menés en atelier afin d'évaluer son incidence sur la surface métallique, ainsi que ses limites tout en se fondant sur des études de cas.

\section{Problématique de conservation des alliages cuivreux dorés}

L'objectif principal de ces interventions de nettoyage est d'éliminer les couches d'encrassement superficielles ainsi que l'irisation qui apparaît en surface de l'or. En effet, les éléments cuivreux dorés ont tendance à s'assombrir avec le temps sous l'action de l'oxygène, des polluants atmosphériques et de l'humidité ${ }^{1}$. De plus, il s'agit dans certains cas de retirer d'anciens revêtements de restauration vieillis et défectueux. Comme dans le cas des objets en argent verni ${ }^{2}$, la vapeur d'eau chaude permet d'obtenir un résultat satisfaisant sur les bronzes dorés.

Une problématique particulière est le traitement de géométries de surface parfois très complexes et le respect des contrastes entre les parties ciselées aux mats et les parties brunies. Le bruni de la dorure, effectué par écrasement de la matière, est un traitement de surface qui permet de créer une profondeur dans les décors de la ciselure. Les zones en retrait des mats sont particulièrement difficiles à atteindre et peuvent être accidentellement polies dans le cadre de traitements mécaniques, même doux, au coton. De plus, un rinçage

Emmanuel Plé, chef de travaux d'art, restaurateur métal, filière Arts décoratifs, département Restauration, C2RMF (emmanuel.ple @ culture.gouv.fr).Julie Schröter, conservatrice-restauratrice de métaux, Paris (julie.schroeter@yahoo.fr). 
fastidieux est nécessaire afin d'éviter d'éventuels problèmes post-traitement liés à la présence de résidus chimiques, par exemple dus à l'emploi de complexants.

\section{Protocole de nettoyage à la vapeur d'eau chaude saturée : principe et avantages du dispositif employé au sein des ateliers}

L'action nettoyante de la vapeur d'eau chaude est générée par la projection, en surface du bronze, d'un fin jet d'eau à l'état gazeux. L'intervention peut être modulée par l'action conjuguée de la température en surface, de la pression, de la forme de la buse ainsi que de sa distance et de son inclinaison par rapport à la surface traitée. L'appareil ${ }^{3}$ est employé à une pression en moyenne de 2,5-3 bars.

En comparaison avec d'autres méthodes de nettoyage (notamment les complexants en milieu aqueux), le recours à la vapeur d'eau chaude comporte un certain nombre d'avantages. L'utilisation d'eau déminéralisée seule en fait un procédé non toxique vis-à-vis de l'utilisateur et permet également de s'affranchir des problématiques de résidus chimiques ou solides à rincer. En effet, certaines méthodes nécessitent d'employer, en concentration variable, des solutions complexantes ou un solvant polaire (éthanol). Ces procédés peuvent avoir des conséquences néfastes pour l'objet, le matériel et l'intervenant. Les résidus de produits toxiques peuvent souiller le réservoir, le conduit d'alimentation et l'extrémité de la buse, et avoir, sur certaines œuvres, des conséquences graves lors d'interventions de nettoyage ultérieures.

L'emploi d'une eau déminéralisée, seule, à température élevée ${ }^{4}$, permet d'obtenir une action dégraissante sans utiliser de détergent ou d'autre agent chimique. De ce fait, une protection particulière des revers des bronzes n'est pas systématiquement requise. En revanche, un démontage des éléments est préconisé afin d'éviter un emprisonnement de l'humidité dans les zones confinées et les porosités de la fonte. Lors du traitement, la présence d'eau sur les revers permet d'éliminer la poussière sans avoir d'incidence sur les traces technologiques. Sur les avers, son action n'affecte pas les mats.

\section{Tests d'évaluation de la température sur la surface en cours de nettoyage}

Dans la mesure où cette technique implique une chauffe de la surface métallique, des essais simples ont été réalisés en atelier afin d'obtenir une idée des températures ${ }^{5}$ pouvant être atteintes en surface d'une plaque en laiton ${ }^{6}$ non doré. Le jet de vapeur statique (buse fine) du générateur de vapeur (GV) est positionné en utilisant quatre modes opératoires différents :

- perpendiculairement au thermomètre ;

- incliné à $45^{\circ}$;

- en balayant la surface de manière linéaire ;

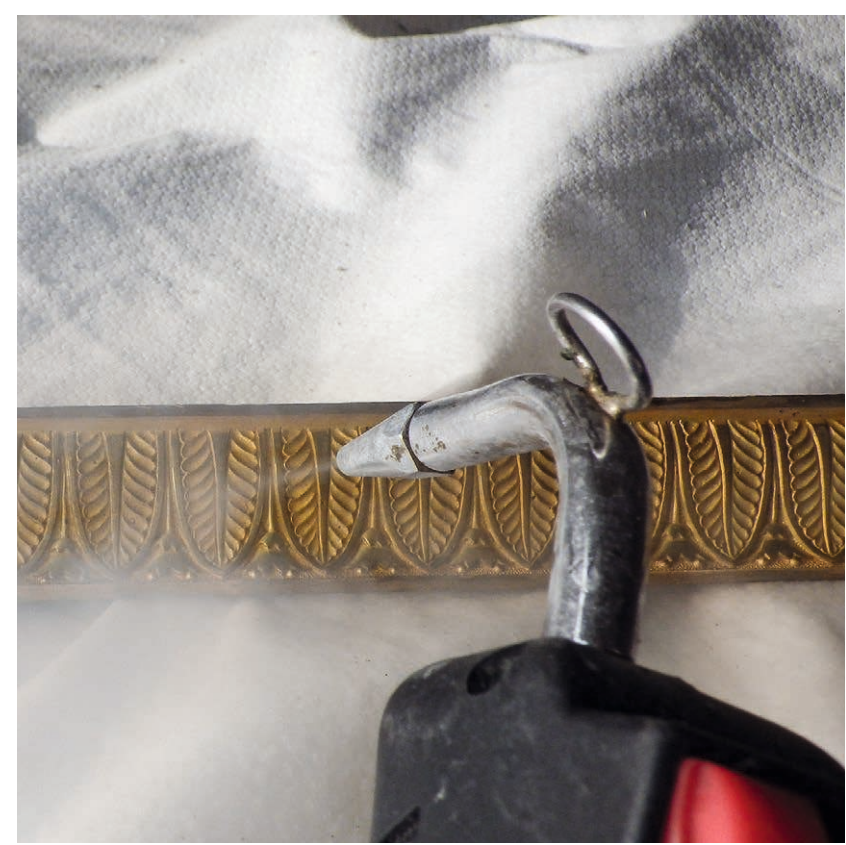

Fig. 1. Nettoyage au générateur de vapeur en balayant circulairement la surface. (C) C2RMF/E. Plé.

- en balayant la surface de façon circulaire.

Les distances de travail observées varient entre 2 et $12 \mathrm{~cm}$, avec des essais d'une durée de 20 secondes chacun.

\section{Résultats}

Le tableau 1 montre que la température du jet entrant en contact avec la surface varie alors entre $42{ }^{\circ} \mathrm{C}$ et $77^{\circ} \mathrm{C}$. De manière générale, on considère que des modifications au niveau métallurgique sont uniquement induites au-delà d'une chauffe de longue durée supérieure à $100{ }^{\circ} \mathrm{C}^{7}$. Les essais démontrent que le type de mise en œuvre de la buse (geste et distance objet/buse) a un impact important sur les températures atteintes. Le passage circulaire de la buse apparaît comme la méthode limitant le plus la chauffe de la surface métallique (fig. 1). Une modification de la couleur du métal en surface n'a pas été observée à l'œil nu.

En outre, cette diffusion de chaleur dans le métal peut potentiellement varier en fonction d'autres facteurs qui n'ont pas été étudiés dans le cadre des essais :

- l'épaisseur de la dorure et son état de surface ;

- la vitesse de passage du jet de vapeur ;

- la géométrie de la buse (diamètre rond ou aplati) ;

- la conductivité thermique du métal (nature du matériau). 
Tableau 1. Mesure des températures du jet de vapeur au contact de la plaque de laiton non doré - récapitulatif des résultats obtenus (températures indiquées en degrés Celsius).

\begin{tabular}{|l|l|l|l|l|}
\hline $\begin{array}{l}\text { Distance } \\
\text { en cm } \\
\text { Durée } 20 \mathrm{~s}\end{array}$ & $\begin{array}{l}\mathrm{GV} \\
\text { perpendiculaire } \\
\text { (statique) }\end{array}$ & $\begin{array}{l}\mathrm{GV} \\
\text { incliné } 45^{\circ} \\
\text { (statique) }\end{array}$ & $\begin{array}{l}\mathrm{GV} \\
\text { linéaire } \\
\text { (en balayage) }\end{array}$ & $\begin{array}{l}\text { GV } \\
\text { circulaire } \\
\text { (en balayage) }\end{array}$ \\
\hline $12 \mathrm{~cm}$ & $48,7^{\circ} \mathrm{C}$ & $46,2^{\circ} \mathrm{C}$ & $43,9^{\circ} \mathrm{C}$ & $42,7^{\circ} \mathrm{C}$ \\
\hline $10 \mathrm{~cm}$ & $54,8^{\circ} \mathrm{C}$ & $50,2^{\circ} \mathrm{C}$ & $47,9^{\circ} \mathrm{C}$ & $45,6^{\circ} \mathrm{C}$ \\
\hline $8 \mathrm{~cm}$ & $56,6^{\circ} \mathrm{C}$ & $55{ }^{\circ} \mathrm{C}$ & $52,8^{\circ} \mathrm{C}$ & $47,5^{\circ} \mathrm{C}$ \\
\hline $6 \mathrm{~cm}$ & $60,3^{\circ} \mathrm{C}$ & $58,6^{\circ} \mathrm{C}$ & $56^{\circ} \mathrm{C}$ & $50,5^{\circ} \mathrm{C}$ \\
\hline $4 \mathrm{~cm}$ & $66,9^{\circ} \mathrm{C}$ & $64,2^{\circ} \mathrm{C}$ & $64,5^{\circ} \mathrm{C}$ & $55{ }^{\circ} \mathrm{C}$ \\
\hline $2 \mathrm{~cm}$ & $77,2^{\circ} \mathrm{C}$ & $71,9^{\circ} \mathrm{C}$ & $75,9^{\circ} \mathrm{C}$ & $75,3^{\circ} \mathrm{C}$ \\
\hline
\end{tabular}

\section{Limitations possibles}

Si cette méthode de nettoyage comporte un certain nombre d'avantages comme la non-toxicité, l'absence de résidus de nettoyage et une élimination des couches de ternissure très efficace sur des parties ciselées au mat, les brunis restent ternes. Il est même possible d'observer un phénomène de voile opaque qui se forme sur ces zones au contact de l'eau déminéralisée chaude, observation souvent faite après le séchage. Cette dernière opération essentielle est exécutée au pistolet à air chaud ou froid.

De plus, il faut être vigilant par rapport aux finitions intentionnelles et aux sensibilités particulières des bronzes dorés (porosité, fragilités structurelles, migration d'éléments $^{8}$ ). À ce titre, on peut citer la mise en couleur de l'or, qui est fréquemment effectuée au XviI ${ }^{\mathrm{e}}$ siècle à l'oxyde de $\mathrm{fer}^{9}$, ainsi que la dorure au mat caractéristique des XviII ${ }^{\mathrm{e}}$ et $\mathrm{XIX}^{\mathrm{e}}$ siècles. La mise en couleur consiste à patiner les dorures en appliquant ou en saupoudrant des sels métalliques (fig. 2), conditionnés en pâte ou en poudre, sur les éléments décoratifs placés sur une grille ou une plaque au-dessus d'un foyer. La chaleur migre dans le substrat qui monte en température. Les sels se liquéfient et se diffusent sur ou dans la dorure, d'où cette particularité chromatique dégradée sur les côtés

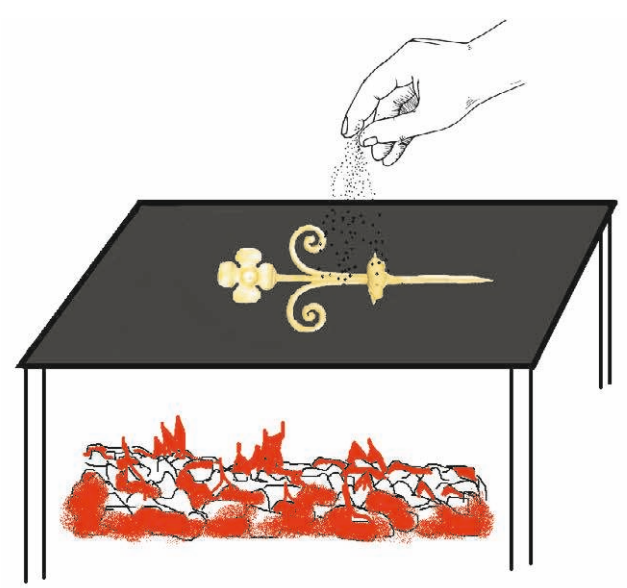

Fig. 2. Représentation schématique du procédé de mise en couleur appliqué à une pièce en alliage de cuivre doré. (C) C2RMF/E. Plé.

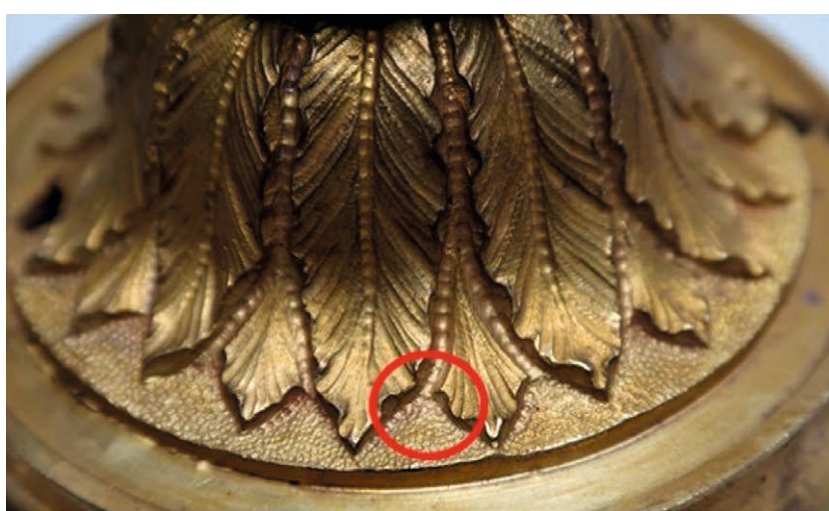

Vue d'ensemble

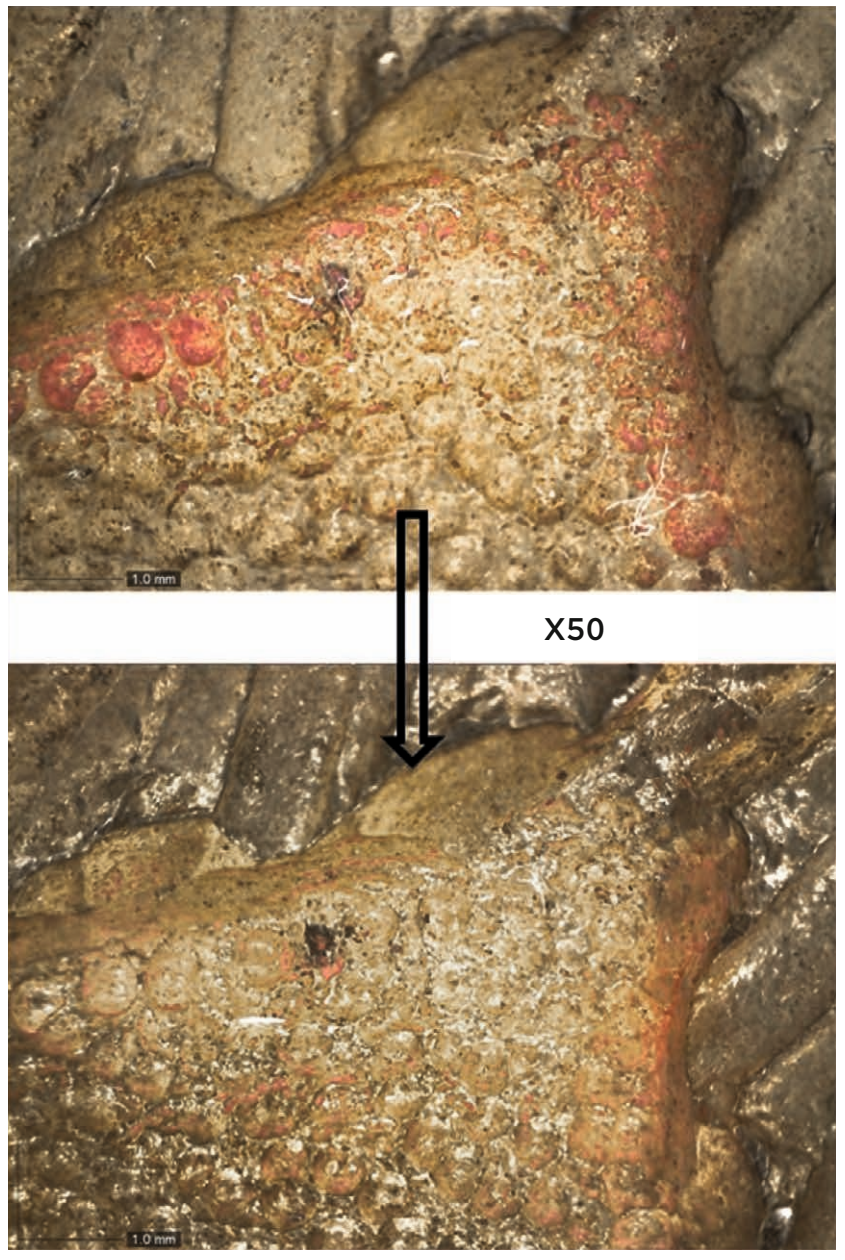

119

Fig. 3. Vue de détail d'un bronze démonté de la pendule Le Jour et la Nuit (inv. CA 1370), musée des Beaux-Arts de Dijon ; zone d'essai en microscopie optique avant (haut) et après nettoyage (bas). (C) J. Schröter. 
des bronzes. À l'issue de cette opération, l'artisan retire par brossage les excédents de sels brûlés afin de contrôler la coloration de la dorure. Avec la méthode de nettoyage étudiée, une chauffe trop importante peut engendrer une altération des sels métalliques, une disparition partielle ou complète de la patine intentionnelle.

Les procédés et la sensibilité des mises en couleur étant variés, tout comme l'évolution des objets dans le temps, un systématisme concernant la méthode de nettoyage doit être à tout prix évité.

La pertinence d'un nettoyage à la vapeur d'eau chaude dépend de la nature, de la solubilité et de l'adhésion des composés présents en surface, comme le montre l'exemple de la pendule Le Jour et la Nuit (inv. CA 1370), datant de 1728 et conservée au musée des Beaux-Arts de Dijon (fig. 3). Grâce aux observations menées dans le cadre du projet de conservation-restauration, la présence d'une mise en couleur des bronzes dorés sous forme de matière rougeâtre dans les aspérités de surface de la dorure a été identifiée. L'étude des bronzes nous a permis de démontrer que cette dernière était probablement postérieure à la date de fabrication de l'objet, et issue d'un remaniement de la surface dorée. Les tests de nettoyage effectués sur ces bronzes ont conduit à déterminer qu'une distance trop faible entre la buse et la surface $(<4 \mathrm{~cm})$ et, de ce fait, une pression et une chauffe plus importantes pouvaient fragiliser localement ce type de finition dans les zones les moins adhérentes. Dans ce cas précis, on observe en microscopie optique, sur des zones d'essai, des pertes de matière colorante rouge après nettoyage.

Enfin, il convient de rappeler que certains bronzes, malgré leur aspect doré, n'ont été recouverts que d'une couche de vernis afin d'imiter une dorure véritable ${ }^{10}$. Ce type de finition pourrait être irrémédiablement dégradé par un nettoyage à la vapeur d'eau chaude. Toute intervention doit donc être précédée d'un diagnostic approfondi.

\section{Principe et mise en ouvre du gommage : sublimation des brunis}

Comme évoqué plus haut, le nettoyage à la vapeur d'eau chaude ne permet pas d'obtenir un résultat satisfaisant sur les brunis. De ce fait, une intervention complémentaire de nettoyage est requise, limitée aux reliefs nécessitant une action plus intense. Cette étape est effectuée avec une gomme Staedler ${ }^{\circledR}$.

Dans le cadre du traitement d'une paire de consoles de Jacob-Desmalter et Thomire (inv. F 3402 et F 170 C), datées de 1809 et conservées au château de Fontainebleau, trois mises en œuvre ont été testées sur la dorure. La gomme a été manipulée de trois manières différentes : de manière verticale, longitudinale et circulaire. C'est ce dernier mode opératoire qui semble donner visuellement un bon résultat esthétique (fig. 4). Il permet d'intervenir de manière précise et contrôlée, et la brillance obtenue est plus homogène. Le caractère faiblement abrasif de cette méthode a été confirmé par le travail réalisé dans le cadre d'un mémoire sur l'évaluation de l'impact des gommes et éponges employées sur les métaux dorés ${ }^{11}$. Des résidus de gommage sont certes constatés, mais peuvent être éliminés facilement par micro-aspiration ou à l'aide d'un pinceau doux. Par mesure de précaution, il est souhaitable de dégraisser au solvant la surface en fin d'intervention. Cette ultime étape peut être réalisée à l'aide de ligroïne 100/140 et d'acétone.

\section{Conclusions et perspectives de développement}

En pratique, le nettoyage des bronzes dorés réalisé à la vapeur d'eau chaude, suivi d'un gommage des brunis, comporte certains avantages (faible toxicité, limitation des risques de résidus de nettoyage...). Toutefois, cette méthode nécessite d'ajuster un certain nombre de paramètres en fonction de la sensibilité des surfaces. Les essais menés en atelier sur des bronzes non dorés avec le générateur de vapeur d'eau chaude ont démontré que les températures atteintes avec différentes mises en œuvre de la buse sont toujours largement inférieures à $100{ }^{\circ} \mathrm{C}$. Un passage circulaire de la buse permet de limiter la hausse de température en surface du métal. Pour compléter cette étude, il serait intéressant d'approfondir la question de l'effet thermique, notamment la possible oxydation des surfaces, grâce à des essais complémentaires effectués cette fois sur des échantillons dorés, et de procéder à une évaluation des possibles modifications par des mesures de couleur. Enfin, il apparaît essentiel de vérifier l'impact éventuel de ce procédé sur des finitions telles que des mises en couleur par un examen à échelle microscopique.

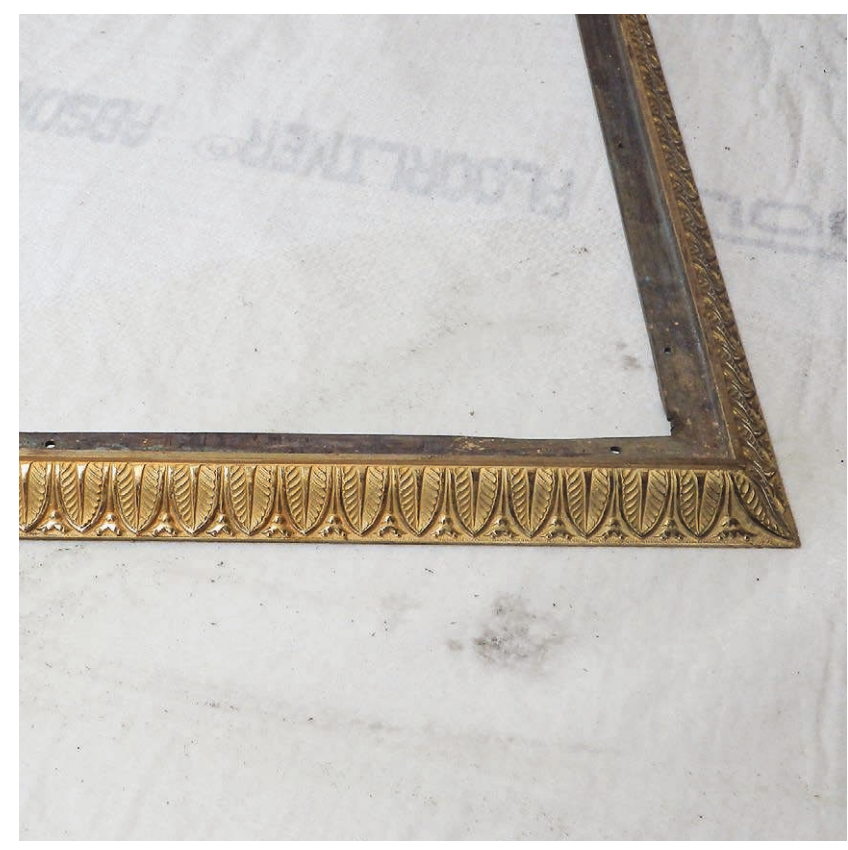

Fig. 4. Sublimation des brunis par gommage circulaire. (c) C2RMF/E. Plé. 
Les essais conduits permettent d'envisager des pistes d'amélioration, comme un dispositif visant à mieux contrôler le processus de nettoyage en temps réel, notamment en maîtrisant le paramètre thermique et les possibles variations de chaleur en surface du métal (via un système d'alerte par exemple). Les mises au point pourraient être affinées par l'ajout d'un curseur de réglage de la pression - à l'instar de ce qui existe pour la pratique du microsablage - et la mise à disposition d'un jeu de buses à géométries/extrémités plus variées. Ces outils supplémentaires permettraient d'adapter la diffusion de la vapeur aux surfaces présentant des patines intentionnelles. Dans cette même perspective, la mise en place d'un capteur vidéo miniaturisé à l'extrémité du diffuseur de vapeur et relié à un écran numérique pourrait être un moyen de contrôle efficace pour le nettoyage de toute surface.

\section{Notes}

1. Scott, 2002, p. 232.

2. Ankersmit, Langh, 2002, p. 1.

3. Modèle Minor ${ }^{\circledR} 164$, fournisseur CTS.

4. À partir de $40^{\circ} \mathrm{C}$, l'eau chaude facilite la dissolution des graisses et des huiles.

5. La température est mesurée grâce à un thermomètre électronique en contact avec la plaquette d'essai en laiton.

6. Laiton industriel courant non oxydé et de finition polie.

7. Volfovsky, 2001, p. 40.

8. Robcis, Thomas, Plé, 2018, p. 91.

9. Pappot, 2014, p. 33

10. Verlet, 1987, p. 176.

11. Lafitte, 2017.

\section{Bibliographie}

Ankersmit H. A., Langh van R., 2002, "The removal of lacquers from silver by steam", in J. A Mosk., N. H. Tennent (eds.), Contributions to conservation: research in conservation at the Netherlands Institute for Cultural Heritage (ICN), Earthscan Ltd., Londres, p. 1-9.

Pappot A., 2014, "Mis en couleur: the coloring of gilt bronze: a review of recipes", in M. Vasques Dias (ed.), Furniture finishes: past, present and future of transparent wood coatings. Proceedings of 12th International Symposium on wood and furniture conservation: Stichting Ebenist,

Amsterdam, 14-15 November 2014, Stichting Ebenist, Amsterdam, p. 30-39.

Robcis D., Thomas C., Plé E., 2018, « Constat d'états de surfaces : les patines intentionnelles sur argent et or ", dans L. Mailho et al. (dir.), Les préalables à la restauration. Actes des Journées d'étude,
13-14 octobre 2016, musée des Beaux-Arts de Nancy, C2RMF, p. 91-104 [http:// technologies.c2rmf.fr/c2rmf/ JDNNancy_C2RMF.pdf].

Scott D., 2002, Copper and bronze in art: Corrosion, Colorants, Conservation, Getty Publications, Los Angeles.

Verlet P., 1987, Les bronzes dorés français du $X V I I I^{e}$ siècle, Éditions Picard, Paris.

Volfovsky C., 2001, La conservation des métaux, CNRS Éditions, Paris.

Document inédit

Lafitte A., 2017, "La cloison d'or ». Étude et conservation-restauration d'un Qilin en émail cloisonné provenant du musée des Arts décoratifs de Paris (XVIII ${ }^{e}$ siècle). Évaluation de l'impact des gommes et éponges pour le nettoyage des cloisons dorées. Mémoire de fin d'étude, Institut national du patrimoine, Paris. 